\title{
Two-Level Multi-criteria Model for Calculating Multinomenclature Spare Parts of an Auto Service Enterprise Based on the Rougher Algorithm for Optimizing the Behavior of Their Particles
}

\author{
Karimov Nijat Ashraf ${ }^{1}$, Dyshin Oleq Aleksandr², Gozalov Sulhaddin Kamal ${ }^{1}$ \\ ${ }^{1}$ Department of Automotive Engineering, Azerbaijan Technical University, Baku, Azerbaijan \\ ${ }^{2}$ Department of Applied Mechanics, Azerbaijan State University of Oil and Industry, Baku, Azerbaijan \\ Email address: \\ nicat.kerimov12@gmail.com (K. N. Ashraf),oleg.dyshin@mail.ru (D. O. Aleksandr),sssz@mail.ru (G. S. Kamal)
}

\section{To cite this article:}

Karimov Nijat Ashraf, Dyshin Oleq Aleksandr, Gozalov Sulhaddin Kamal. Two-Level Multi-criteria Model for Calculating Multinomenclature Spare Parts of an Auto Service Enterprise Based on the Rougher Algorithm for Optimizing the Behavior of Their Particles. Science Research. Vol. 5, No. 4, 2017, pp. 57-64. doi: 10.11648/j.sr.20170504.12

Received: August 29, 2017; Accepted: September 18, 2017; Published: November 8, 2017

\begin{abstract}
On the example of the two-criterion problem with the objective functions of the maximum, the confidence probabilities of the demand and the minimum of the total costs show the applicability of the method of Vector Optimization of Particle Swarm Optimization (VEPSO). Compared with genetic algorithms and other methods of evolutionary modeling, this method is easy to implement and has high efficiency, as well as the accelerated cost of an approximate solution of the problem from the external archive of the no dominant best solutions to the Pareto front, which is the boundary of the Pareto-optimal Compromise) solutions.
\end{abstract}

Keywords: Rougher Algorithm, Swarm Optimization, Vector Optimization of Particle Swarm Optimization (VEPSO)

\section{Introduction}

In recent decades, the problem of managing the resources of spare parts is becoming increasingly important. The reason for this is the increased requirements for quality and reliability of servicing complex technical systems. The task of placing spare parts at different levels of supply is to achieve maximum integrated benefits and guarantee high efficiency of technical training of the serviced systems. Despite numerous studies of this problem (see, for example, [1-3]), in most papers only one criterion has been optimized. In fact, the task of placing spare parts for maintenance companies should be formulated simultaneously for several target functions, as well as the required probability of sufficient volume of the order of spare parts for the planned period and minimization of total costs.

Particle swarm optimization (PSO) is a kind of evolutionary computational technology based on the theory of the propensity of particles (ants, bees, birds, etc.) to construct a column, groups, and proposed by Eberhart and
Kennedy [4] in 1965 The idea of the PSO-method is to simulate the behavior of birds when hunting for food. In this method, it is assumed that a group of birds reaches a food source randomly, while the food is in some specific area. The optimal strategy for finding food is to reach the nearest neighborhood of food from any current position of the birds. In the PSO-method, each individual unit is taken as a particle with a certain position $(x)$ and $(v)$ speed. The solution of the problem is the optimal position $\left(x^{*}\right)$ of the particles.

The collective system is able to solve complex dynamic tasks for performing collaborative work that could not be performed by each element of the individual system in a variety of environments without external management, control, or coordination. In such cases, talk about swarm Intelligence, as a special way of cooperative behavior, that is, survival.

Among the so-called "Soft computing techniques", 
developed over the past 50 years for difficult to solve discrete optimization problems, are divided: genetic algorithms (GA) [5-8], based on natural selection and genetics; Ant (Art Colony Optimization - ACO, Art Systems - AS), modeling the anthill's behavior [9-12].

Unlike the PSO method, the multi-objective particle swarm optimization (MOPSO) method is formulated for several objective functions and makes a choice from a set of possible solutions. The key issue here is the appropriate choice of the fitness function, which measures the quality of the multi-criteria problem solving scheme. In accordance with the choice of the fitness function, the solution schemes in the PSO method are classified as: target polymerization method, Pareto dominance method, and rule-based method. The first of them is related to the polymerization of several objective functions by adding their degrees and invoking several objective functions into one objective function [13, 14]. The Pareto-dominance method [15-17] best selects a good solution providing a global mechanism, which guides the MOPSO method to find a good solution with a symmetric distribution. In the method based on the rule [18, 19], not all objective functions are considered simultaneously. According to various limitations of the optimization problem, the fitness function is constructed according to the objective functions chosen in accordance with the rule.

In [20], an improved MOPSO method for implementing spare parts in the multi-criteria optimization model was proposed, which provides the maximum confidence in the chosen amount of spare parts and the minimum costs accepted as objective functions. Dimension reduction and multicriteria optimization are used to increase the effectiveness of the MOPSO method.

\section{Formulation of the Problem}

Consider a spare parts management system consisting of two echelons. The first echelon is a firm auto service company (AC), engaged in the repair and maintenance of cars, and the second echelon - the supply center for spare parts (parts) of these cars. Spare parts differ in the standard: engine $(i=1)$, body $(i=2)$ and $\operatorname{suspension}(i=3)$.

In contrast to [20], we will consider in the first echelon one type of service (equipment), in our case it is a car and one serving $j$ - th inventory (inventory of the first echelon). As in [20], it is believed that the first echelon acquires (purchases) the necessary spare parts in the same local inventory center (inventory of the second echelon). The task of placing deliveries of spare parts from the second echelon to the first will be solved under the following assumptions:

1) The demand for each type of vehicle parts is subject to one of six parametric distributions (exponential $(j=1)$, normal $(j=2)$, lognormal $(j=3)$, We bull $(j=4)$, diffusion-free no monotonic $(j=5)$, diffusion monotonic $(j=6))$;

2) Each of the parts of the car is of equal importance and the failure of one of them causes the failure of the car itself; The second echelon supplies spare parts to a set of first-tier inventories, including the $\mathrm{AC}$ under consideration, with all first-tier inventories having roughly the same average demand and the same maximum allowable levels for each type of spare parts.

3) Each of the failed parts is replaced respectively with a spare part and sent to a repair base;

4) The second echelon supplies spare parts to a set of first-tier inventories $\left\{\mathrm{ACP}_{\mathrm{m}}\right\},\left(m=1, \ldots, m_{0}\right)$, including the $\mathrm{AC}$ under consideration, with all first-tier inventories having roughly the same average demand $\mathrm{E}_{i, m}\left(m=1, \ldots, m_{0}\right)$ and the same maximum allowable levels $\mathrm{S}_{i, m}^{\max }\left(m=1, \ldots, m_{0}\right)$ for each type of $i$ - th spare parts.

To solve the problem, the following notations are used:

$i$ - number of the type of spare parts;

$n$ - the total number of types of spare parts (in our case $N=3$ );

$T_{\mathrm{PS}}$ - period of replenishment of spare parts in the first echelon;

$T_{m}$ - period of replenishment of spare parts in the second echelon;

$E_{i}$ - average demand for spare parts of the first type in the first echelon in the previous period; $T_{\text {non }}$;

$S_{i}$ - the required (to be determined) number of $i$-th type spare parts in the first echelon in the planned period $T_{\text {non }}$

$S_{o i}$ - number of spare parts of the $i$ - th type in the second echelon in the planned period;

$E\left[B\left(S_{i}\right)\right]$ - expected deficit in spare parts in the first echelon for a fixed value $S_{i}$;

$E\left[D\left(S_{i}, S_{o i}\right)\right]$ - expected deficit in spare parts of the $i$-th type in the second echelon at fixed values $S_{i}$ and $S_{o i}$;

$x_{i}$ - the actual value of the accumulated demand for $i$ - th type spare parts in the first echelon at some point in time from the previous period $T_{\text {non }}$;

$P\left(x_{i}\right)$ - probability of the accumulation of demand for spare parts of the $i$-th type in the first echelon at some point in time from the previous period $T_{n o n}$;

$\xi_{i}$ - the probability of a deficit in spare parts of the $i$ - th type in the second echelon at fixed values $S_{i}$ and $S_{o i}$;

$\eta_{i}$ - probability of a deficit in spare parts of the first $i$-th type in the first echelon with a fixed $S_{i}$;

$S_{i}^{\max }$ and $S_{o i}^{\max }$ - the maximum permissible level of spare parts of the first $i$ - th type in the first and second echelon, respectively;

$x_{i}^{\prime}$ - the amount of deficit in spare parts of the first $i$ - th type in the first echelon for a fixed $S_{i}$; 
$E_{o i}$ - average demand for spare parts of the $i$-th type in the second echelon;

$C_{i}$ - spare part price of $i$-th type;

$T_{o i}$ - average time between failures (average operating time) of parts of the $i$-th type.

\section{Preparatory Part of the Model}

(1) Expected demand for spare parts in the first echelon.

The expected value of the demand for $i$-th type spare parts in the first echelon is defined

$$
E_{i}=\sum_{k_{1}}^{k_{0}} x_{i, k} \cdot P\left(x_{i, k}\right)
$$

where is the $\left\{x_{i, k}\right\}$ sequence of accumulated failures of the car $i$ - th type parts belonging to the cars, received for maintenance in the considered AC during the first $k$ working day from the previous replenishment period consisting of $k_{0}$ working days.

Here $P\left(x_{i, k}\right)=\left.\frac{d}{d x} F_{j_{\text {dem }, i}^{*}}(x)\right|_{x=x_{i, k}}$, where $F_{j_{\text {dem }, i}^{*}}$ is the best (in the sense of greatest consistency with the empirical distribution function) distribution function from the above list of six theoretical parametric distributions. For the particular example considered in the previous paper [21], specific example $j_{d e m, 1}^{*}=5, j_{d e m, 2}^{*}=5, j_{d e m, 3}^{*}=4$

The approximate value for $P\left(x_{i}\right)$ can be determined from formula

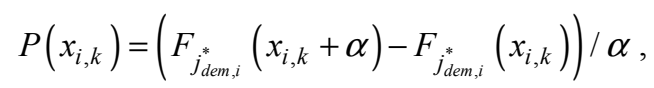

Where: $\alpha$ is a sufficiently small positive number.

(2) The relative shortage ratio in spare parts in the first echelon.

The expected deficit in type-I spare a part in the first echelon is defined as

$$
E\left[B\left(S_{i}\right)\right]=\sum_{x_{i}=S_{i}+1}^{\infty}\left(x_{i}-S_{i}\right) \cdot P\left(x_{i}\right)
$$

Expected relative deficit, i.e. the probability of a $i$-th type deficit in the first echelon is defined as

$$
\eta_{i}=\frac{E\left[B\left(S_{i}\right)\right]}{E_{k}} .
$$

(3) Relative shortage ratio of spare parts in the second echelon.

The situation when the actual demand in spare parts of the $i$-th type exceeds the level of their stock in the first echelon, necessitates an additional order sent to the second echelon. Consequently, the probability of demand for spare parts of the $i$-th type in the second echelon $P_{0}\left(x_{i}^{\prime}\right)$ is defined as

$$
P_{0}\left(x_{i}^{\prime}\right)= \begin{cases}P\left(x_{i}^{\prime}+S_{i}\right) & \text { при } x_{i}^{\prime}>0, \\ \sum_{x_{i}=0}^{S_{i}} P\left(x_{i}\right) & \text { при } x_{i}^{\prime}=0 .\end{cases}
$$

On the basis of (4), the expected demand for spare parts of the $i$-th type in the second echelon

$$
E_{o i}=\sum_{y_{i}=0}^{\infty} y_{i} \cdot P_{0}\left(y_{i}\right) \text {. }
$$

Expected replacement parts deficit $i$-th type in the second tier is defined as

$$
E\left[D\left(S_{i}, S_{o i}\right)\right]=\sum_{y_{i}=S_{o i}+1}^{\infty}\left(y_{i}-S_{o i}\right) \cdot P_{0}\left(y_{i}\right)
$$

and the probability of a deficit in spare parts of the $i$-th type in the second echelon is represented in the form

$$
\xi_{i}=\frac{E\left[D\left(S_{i}, S_{o i}\right)\right]}{E_{o i}}
$$

In the absence of data on $S_{o i}$ and $E_{o i}$, we shall assume $\xi_{i}$ that it is small, for example $\xi_{i}=0,05$.

(4) Average delay time for receipt of spare parts in the first echelon.

The delay in the receipt of spare parts in the first echelon occurs in two cases. In the first case, when there is a deficit in spare parts in the first echelon, while in the second echelon there is a sufficient number of these spare parts. Then the arrival delay time is expressed in the form $\left(1-\xi_{i}\right) \cdot \eta_{1} \cdot T_{\text {non }}$. In the second case, when there is a deficit in spare parts both in the first and second echelons. Then the arrival delay time is $\eta_{1} \cdot \xi_{i} \cdot\left(T_{n o n}+T_{m}\right)$. Consequently, the average delay in the receipt of spare parts of the first $i$ - type in the first echelon is presented as:

$$
T_{o i}=\left(1-\xi_{i}\right) \cdot \eta_{1} \cdot T_{\mathrm{PZ}}+\xi_{i} \cdot \eta_{1}\left(T_{\mathrm{PZ}}+T_{m}\right)=\eta_{1} \cdot T_{\mathrm{PZ}}+\xi_{i} \cdot \eta_{1} \cdot T_{m}
$$

(5) Confidence in meeting the demand for spare parts of the $i$-th type

Statistical modeling of demand is carried out on the basis of failure statistics in the previous planning period. The identification of the best model of demand (or, what is still, the model of accumulated failures) and the mean time between failures were investigated by us in the previous paper [21].

In order to determine the average time between failures of $i$-th type parts $\left(T_{o i}\right)$ in [21], the following random variables (briefly r.v.) are introduced into consideration: r.v. operating time $(T)$ to failure with values $t$ and r.v. Operating time 
before the $r$-th failure $\left(T_{r}\right)$ with values $t_{r} ;$ r.v. the number of failures $(R)$ with values $r$ and $R_{r_{0}}-$ r.v. with values $r \leq r_{0}$, where $r_{0}$ - is some fixed number. In this case, the relations $\mu_{T_{r_{0}}}=T_{0} \cdot \mu_{R_{r_{0}}}$ and $v_{T_{r_{0}}}=v_{R_{r_{0}}}$ are satisfied, where $\mu$ and $v$ is the mean value and variance of the r.v., and between the distribution functions of the quantities

$$
\begin{aligned}
& F_{T_{r_{0}}}\left(t ; \mu_{T_{r_{0}}}, v_{T_{r_{0}}}\right)=\operatorname{Bep}\left(T \leq t \mid r \leq r_{0}\right)= \\
& F_{T_{r_{0}}}\left(t ; \mu_{R_{r_{0}}} \cdot T_{0}, v_{T_{r_{0}}}\right)=F_{R_{r_{0}}}\left(r ; \mu_{R_{r_{0}}}, \nu_{R_{r_{0}}}\right)
\end{aligned}
$$

The value $T_{o i}$ of the quantity is found from the solution of equation

$$
F_{T_{r_{i, k_{0}}}}=\left(T_{\mathrm{PZ}}\right)=\alpha_{i}
$$

$r_{i, k_{0}}^{\mathrm{H}}$ - Cumulative number of failures by the end of the period $T_{\mathrm{PZ}}\left(k=k_{0}\right) ; \alpha_{i}=\pi_{\partial i}^{m p}$

( $\pi_{\partial i}^{m p}$ - indicator of the adequacy of providing nonrenewable parts of the $i$-th type).

In the case of Weibull distribution $\left(j_{d e m, 3}^{*}\right)=4$, we have

$$
T_{0,3}=\frac{T_{\mathrm{PZ}} \cdot \Gamma\left(1+v_{R_{r_{3}^{\mathrm{H}}}}\right)}{\mu_{R_{r_{3, k_{0}}^{\mathrm{H}}}} \cdot\left[\ln \left(\frac{1}{1-\alpha_{3}}\right)\right]^{1 / v_{R_{r_{3}^{\mathrm{H}}}}}},
$$

where: $\Gamma(x)$ is the gamma function.

In the case of a diffusion nonmonotonic distribution $\left(j_{d e m, 1}^{*}\right)=\left(j_{d e m, 2}^{*}\right)=5$, the above equation for finding $T_{o, i}$ $(i=1$ и $\mathrm{i}=2)$ can be written in the form of a nonlinear equation $f(x)=0$, wherev $x=T_{o, i}, f(x)=F_{T_{q_{1}+1}}\left(T_{\mathrm{PZ}}\right)-\alpha_{i}$. This equation is solved by Newton's method using the iterative procedure

$$
x_{m+1}=x_{m}-\frac{f\left(x_{m}\right)}{f^{\prime}\left(x_{m}\right)} .
$$

As an initial approximation, one of two simple estimates is taken for $T_{o, i}$ :

$$
T_{o, i}^{(1)}=\frac{1}{k_{0}} \sum_{k=1}^{k_{0}} \frac{1}{r_{i, k_{0}}}, T_{o, i}^{(2)}=\frac{1}{k_{0}} \sum_{k=1}^{k_{0}} \frac{k}{r_{i, k}^{\mathrm{H}}} .
$$

Then the steady-state confidence probability of the spare parts of the $i$-th type in the first echelon will be written in the form:

$$
P_{i}=\frac{T_{o i}}{T_{o i}+T_{D i}}
$$

Multiplying the numerator and the denominator on the right-hand side of (10) by $E_{i}$, taking (2) and (3) into account, we obtain

$$
P_{i}=\frac{T_{o i} \cdot E_{i}}{T_{o i} \cdot E_{i}+\sum_{x_{i, k} \geq S_{1}+1}\left(x_{i, k}-S_{i}\right) \cdot P\left(x_{i, k}\right)\left(T_{n o n}+\xi_{i} \cdot T_{m}\right)}
$$

Therefore, the confidence probability (support probability) of meeting the demand for all spare parts takes the form:

$$
P_{s}=\prod_{i=1}^{n} P_{i}
$$

(6) Multi-criteria model of location and optimization.

Taking into account $S_{o i}=m_{0} \cdot S_{i}$ the relationship, the problem of allocation and optimization of spare parts can be written in the form:

$$
\begin{gathered}
\max _{s_{1}, \ldots, s_{l}} P_{s}=\prod_{i=1}^{n} \frac{T_{o, i} \cdot E_{i}}{T_{o, i} E_{i}+\sum_{x_{i, k} \geq S_{i}+1}\left(x_{i, k}-S_{i}\right) \cdot P\left(x_{i, k}\right) \cdot\left(T_{n o n}+\xi_{i} \cdot T_{m}\right)} \\
\max _{s_{1}, \ldots, S_{l}} C_{s}=\sum_{i=1}^{n} C_{i}\left(1+m_{0}\right) \cdot S_{i} .
\end{gathered}
$$

with restrictions:

$$
\begin{gathered}
0 \leq \frac{\sum_{x_{i, k} \geq S_{i}+1}\left(x_{i, k}-S_{i}\right) \cdot P\left(x_{i, k}\right)}{E_{i}} \leq 1, \quad \forall i=1, \ldots, n . \\
0 \leq S_{i} \leq S_{i}^{\max }, S_{i} \text { - integer, } \forall i=1, \ldots, n .
\end{gathered}
$$

Equations (13) and (14) represent an optimization criterion, the first of which maximizes the confidence probability of satisfying demand, and the second maximizes the cost of realizing the demand for spare parts. Restrictions (15) mean that the relative deficit in spare parts of each type is not less than zero and not more than one.

Restrictions (16) mean that the number of spare parts of each type in the first echelon in the planned replenishment period should not exceed its maximum permissible limit value.

\section{The Method of Multicriteria Optimization of Particle Swarm Behavior (Multi-Objective Particle Swarm Optimization Method-MOPSO-Method)}

The MOPSO method, or as it is also called the Vector 
Evaluated PSO (VEPSO) method, is proposed by Parsopoulos and Vrahatis [14, 23]. This method of the swarm approach to the multicriteria problem is based on the idea of the genetic algorithm for solving the multicriterion problem (Vector Evaluated Genetic Algorithm - VEGA), proposed by Schaffer [24].

The essence of MOPSO-method is the following:

Let the optimization problem consist of $k$ objective functions.

Then, to solve the problem, $k$ swarm populations consisting of particles are $\mu$ - used (in our case these are arbitrary parts of the car, considered independently of their type nomenclature).

Instead of the index $i$ usually used to denote particles $\mu$, we will use the index for their designation, leaving the index to denote the type of particles.
Each swarm $s$-population $(s=1, \ldots, k)$ characterizes the $\mu$-particle as a point in a set of $S n$-dimensional space $x_{\mu}^{[s]}=\left(x_{\mu 1}^{[s]}, x_{\mu 2}^{[s]}, \ldots, x_{\mu n}^{[s]}\right)$, the velocity of a $\mu$-particle in the $s$ - swarm population is denoted as a vector $v_{\mu}^{[s]}$ with components $v_{\mu i}^{[s]}(i=1, \ldots, n) \quad, \quad$ and denotes $p_{\mu}^{[s]}=\left(p_{\mu 1}^{[s]}, p_{\mu 2}^{[s]}, \ldots, p_{\mu n}^{[s]}\right) \in S$ the best position that a point necessarily visits $x_{\mu}^{[s]}, p_{\mu i}^{[s]}$-a vector with components $p_{\mu i}^{[s]}(i=1, \ldots, n)$.

Then the process of vector $x_{\mu}^{[s]}$ correction is represented by an iterative scheme

$$
\begin{aligned}
& v_{\mu i}^{[s]}(t+1)=w \cdot v_{\mu i}^{[s]}(t)+c_{1} r_{1}\left(p_{\mu i}^{[s]}(t)-x_{\mu i}^{[s]}(t)\right)+c_{2} r_{2}\left(p_{g i}^{[q]}(t)-x_{\mu i}^{[s]}(t)\right) \\
& v_{\mu i}^{[q]}(t+1)=w \cdot v_{\mu i}^{[q]}(t)+c_{1} r_{1}\left(p_{\mu i}^{[q]}(t)-x_{\mu i}^{[q]}(t)\right)+c_{2} r_{2}\left(p_{g i}^{[s]}(t)-x_{\mu i}^{[q]}(t)\right) \\
& x_{\mu i}^{[s]}(t+1)=x_{\mu i}^{[s]}(t)+v_{\mu i}^{[s]}(t+1) \\
& x_{\mu i}^{[q]}(t+1)=x_{\mu i}^{[q]}(t)+v_{\mu i}^{[q]}(t+1) \\
& S_{1 \cdot v_{\mu 1}}(t+1)=\omega \cdot S_{1 \cdot v_{\mu 1}}(t)+c_{1} r_{1}\left(S_{1 \cdot p_{\mu i}}(t)-S_{1 \cdot x_{\mu i}}^{\prime}(t)\right)+c_{2} r_{2}\left(S_{2 \cdot p_{g i}}(t)-S_{1 \cdot x_{\mu i}}(t)\right) \\
& S_{2 \cdot v_{\mu 1}}(t+1)=\omega \cdot S_{2 \cdot v_{\mu i}}(t)+c_{1} r_{1}\left(S_{2 \cdot p_{\mu i}}(t)-S_{2 \cdot x_{\mu i}}^{\prime}(t)\right)+c_{2} r_{2}\left(S_{1 \cdot p_{g i}}(t)-S_{2 \cdot x_{\mu i}}(t)\right) \\
& S_{1 \cdot x_{\mu i}}(t+1)=S_{1 \cdot x_{\mu i}}(t)+v_{\mu i}(t+1), 1 \leq \mu \leq N, 1 \leq i \leq n
\end{aligned}
$$

where $w$ - is a positive parameter, called the inertial weight; $c_{1}$ and $c_{2}$ - positive constants, called respectively cognitive and social parameters; $r_{1}$ and $r_{2}$ is the realization of two independent random variables, which are assumed to be uniformly distributed in the interval $(01$,$) . Here p_{g}^{[q]}$ - the best position in the $q$-th swarm population, which is $x_{l} \in N G_{i}, f_{q}\left(p_{g}^{[q]}\right) \leq f_{q}\left(p_{e}^{[q]}\right)$ if the objective function $f_{q}$ is minimized and $f_{q}\left(p_{g}^{[q]}\right) \geq f_{q}\left(p_{e}^{[q]}\right)$ if the objective function is $f_{q}$ maximized. Here $N G_{i} \subseteq S$ is the set of neighbors of a point $x_{i}$, i.e. points $x_{e}$ from a neighborhood of a point $x_{i}$. In formulas (17) - (20) $t$ it means the iteration

In the case of two objective functions, the iteration scheme calculated by the $q$-th objective function, i.e. for all $l \mathrm{c}$,

$$
\begin{gathered}
S_{2 \cdot x_{\mu i}}(t+1)=S_{2 \cdot x_{\mu i}}(t)+v_{\mu i}(t+1), 1 \leq \mu \leq N, 1 \leq i \leq n \\
v_{\mu i}(t+1)=\left[S_{1 \cdot v_{\mu 1}}(t)+S_{2 \cdot v_{\mu 1}}(t)\right]
\end{gathered}
$$

In the MOPSO method, the inertial factor $\omega$ is a very important controlled parameter that is used to control the degree of influence of the current velocity values on its magnitude. The large value of this parameter is more advantageous when conducting a global study, and its small value is in a local study. Numerous experimental studies [25] show that the efficiency of the MOPSO method becomes greatest when the parameter $\omega$ varies between 0.4 and 1.4 In [20], the following formula is used to $\omega$ select the parameter

$$
\omega_{g}=\omega_{\max }-g \frac{\omega_{\max }-\omega_{\min }}{G_{\max }}
$$

where $\omega_{\max }$ and $\omega_{\min }$ is the maximum and minimum inertia weight; $g$ means the number of iterative steps and $G_{\max }$ their maximum number. 


\section{Key Concepts of the MOPSO Method}

(1). Basic definitions and concepts of PSO-methods.

We give the main definitions and concepts used in PSOand MO PSO-methods [22, 25-26].

A multicriterial problem in general form is written as

$$
\min \vec{f}(\vec{x})=\left(f_{1}(\vec{x}), f_{2}(\vec{x}), \ldots, f_{k}(\vec{x})\right)
$$

under restrictions

$$
\begin{aligned}
& g_{i}(\vec{x}) \leq 0, \quad i=1, \ldots, m \\
& h_{j}(\vec{x})=0, \quad j=1, \ldots, p
\end{aligned}
$$

where $\vec{x}=\left(x_{1}, x_{2}, \ldots x_{n}\right)$ is the vector of variables from the $n$-dimensional Euclidean space $R^{n} ; f_{i}: R^{n} \rightarrow R$; $i=1,2, \ldots, k$, are the objective functions of the constraints of the problem.

Definition 1. The best (personal best) position of a particle is the position providing the greatest success, (measured by some scalar quantity analogous to the fitness function used in evolutionary algorithms);

Definition 2. The globally best (global best) position of a $\mu$ particle (denoted $p_{\mu}$ ) is the position providing the greatest success in the neighborhood of this particle, measured by some scalar value, which is determined by the fitness function $\phi(\vec{x})$. As a function $\phi(\vec{x})$, we will use the aggregation of functions $f_{1}(\vec{x}), \ldots, f_{k}(\vec{x})$ with weights $w_{1}, \ldots, w_{k}$ (see formula (30) below) for all particles of the swarm population.

Unlike the one-criterion optimization problem, in which the optimal solution has a clear meaning, in the multicriteria optimization (MO) problem there are many trade-off solutions called Pareto-optimal solutions. Target functions $f_{j}(x)(j=1, \ldots, k)$ in the admissible domain $S \subset R^{n}$ (i.e, the domain in which all $f_{j}(\vec{x})$ functions are defined). Target functions $f_{j}(x)(j=1, \ldots, k)$ in the field $S$ may conflict with each other, so in most cases it is impossible to determine for all functions globally a minimum at the same point. The goal of the MO problem is to construct a set of Paretooptimal solutions.

Definition 3. Let $\vec{u}=\left(u_{1}, \ldots, u_{n}\right)$ and $\vec{v}=\left(v_{1}, \ldots, v_{n}\right)$ be two vectors from $R^{k}$. They say that $\vec{u}$ dominates. $\vec{v}$ (denoted and $\prec \vec{v}$ ) if and only if, when $u_{j} \leq v_{j}, j=1, \ldots, k$, and $u_{j} \leq v_{j}$ at least for one $j$.

This property is called Pareto dominance and is used to determine Pareto-optimal solutions to the MO problem.

Definition 4. It is said that the $\vec{x}$ solution of the Pareto problem is optimal if and only if there is no solution $\vec{u}$ to the one that $\vec{f}(\vec{y})$ dominates $\vec{f}(\vec{x})$. In this case, it is $\vec{x}$ also said that it is nondominant (nondominated) relatively $S$.

Definition 5. The set of all Pareto-optimal solutions of the MO problem is called a Pareto-optimal set and is denoted $\Re^{*}$.

Definition 6. The set

$$
\mathfrak{R} S^{*}=\left\{\vec{f}(\vec{x}) \in \mathfrak{R}^{*}\right\}
$$

called the Pareto - Front.

A Pareto front is a convex set if and only if for all $u, v \in \mathfrak{R} S^{*}$ and all $\lambda \in(0,1)$ there exists $w \in \mathfrak{R} S^{*}$ such that

$$
\lambda\|u\|+(1-\lambda)\|v\| \geq\|w\|
$$

and concave if and only then

$$
\lambda\|u\|+(1-\lambda)\|v\| \leq\|w\|
$$

The Pareto front can also be partially convex and / or concave, as well as discontinuous. The Pareto-optimal set can be infinite. Therefore, the main goal of solving the MO problem is to find as many Pareto optimal solutions as possible that correspond adequately to the Pareto-front range and possibly the least differ from the Pareto front.

Non-dominant best positions $p_{\mu i}$ in (17) - (20) and calculated for each particle towards the global minimum. The definition of leaders does not lead directly to the desired goal, since in the vicinity of the particle there can be many non-dominant solutions, only one of which is usually chosen to correct the speed.

Most trivial solutions can be no dominant and participate as candidates in choosing the best positions of a particle. However, this choice is not always fair, since the size of the Pareto front can exceed the size of the swarm population. Moreover, two no dominant solutions can be equally good, which complicates the problem of unambiguous choice of the best position of the particle. The size problem can be solved by creating an additional set called an external archive to accumulate no dominant solutions obtained in the process of solving problems, since the problem of the correct choice of the members of this archive depends on the approach to its formation. However, the external archive also has a limited size, thus leaving the possibility of building rules for replacing existing solutions with new solutions.

Weighted aggregation of objective functions is the most common approach to constructing the utility function (fitness function)

$$
\phi(x)=\sum_{j=1}^{k} w_{j} \cdot f_{j}(x)
$$

where $w_{j}, j=1, \ldots, k$ are nonnegative scales. It is usually assumed that $\sum_{j=1}^{k} w_{j}=1$. Weights $w_{j}$ can either be fixed or 
adapt in the optimization process.

For the two-criteria MO problem, the following approach to choosing weights is most common

$$
w_{1}(t)=|\sin (2 \pi t / R)|, w_{2}(t)=1-w_{1}(t)
$$

called dynamic weighted aggregation (DWA). Here is the iteration $t$ - index and $R$ is the weighted change in frequency. In the calculations it is usually assumed $R=200$ [27].

The general scheme for solving the MO of the PSOproblem can be written in the form of the following pseudocode [22]:

Begin

Initialize swarm, velocities and best positions

Initialize external archive (initially empty)

While (shopping criterion not satis fied) Do

For each particle

Select a number of the external archive (if needed)

Update velocity and position

Evaluate new position

Update best position and external archive

End for

End While

End

An integer solution of problem (13) - (16) is obtained by rounding up to the nearest integers the values of the component of optimal solutions in the real space $R^{n}$.

Experimental studies have shown [28] that the VEPSO algorithm, described by formulas (17) - (20) in combination with an external archive, tends, in the limit, to the Pareto front.

The strict convergence of the MOPSO method was proved under the following conditions [26]:

1. Decisions included in the external archive at the $(t+1)$ -th iteration should be non-dominant with respect to the solutions generated for all iterations $\tau, 0 \leq \tau \leq t+1$ (monotonicity condition).

2. The algorithm should be able to generate a solution in the neighborhood of the optimal point with non-zero probability for any solution $x$ from the admissible domain.

\section{Conclusion}

The work shows the implementation of the swarm approach to solving the two-level problem of allocations and optimizing the supply of the auto service enterprise (the first echelon) with multi-nomenclature spare parts from the same supply center (second echelon) with weakened assumptions on the distribution of demand and failure of each type of vehicle parts. Namely, in contrast to [20], in which it is assumed that the demand for parts is subject to exponential distribution and their refusals to the Poisson distribution, we consider the case where the distribution of demand and failure of parts is one of the six known theoretical parametric distributions: exponential, Normal, lognormal, We bull, diffusion no monotonic and diffusion monotonic. The last two types of distribution are particularly important for the failure of parts, since any (even minor) disruption of the performance of a particular vehicle component influences the diffusion mode on the performance of other parts. Moreover, replacing a failed part with a spare part cannot completely regenerate the normal functioning of the serviced vehicle

The method of multi-objective optimization of particle behavior (multi-objective particle swarm optimization method - MO PSO-method). In comparison with genetic (HA) and other algorithms of evolutionary modeling, it showed its effectiveness and a sufficiently high confidence probability, providing a rather rapid convergence of the solution to the Pareto front, which is the boundary of the Pareto-optimal solution domain. The implementation of the method is shown on the example of a two-criterion problem that maximizes the confidence probability of meeting the demand and minimizes the total costs for the fulfillment of the planned multi-item spare parts in the replenishment period.

The proposed method for solving the MO problem can be extended to other types of distribution of demand and failure of equipment parts, several maintenance companies that purchase spare parts from equipment from several service centers that are part of a single transport and logistics system (TLS) in the region and can be used in other industries.

\section{References}

[1] Doc. Palmer Maintenance planning and scheduling handbook. New York: Mc Grow Hill Press, 2006.

[2] Duy Quang Nquyen, Bagajewicz Miguel. Optimization of preventive maintenance scheduling in processing plants. // Computer Aided Chemical Engineering, 2008; (25): 319-324.

[3] Ilgin M. Ali, Tenali Semra. Paint optimization of spare parts inventory and maintenance policies using genetic algorithms // International Journal of Advanced Manufacturing Technology 2007; 30 (5): 594-604.

[4] Eberhart R. C. and Kennedy J. A. New Optimizer Using Particle Swarm Theory // Proceedings Sixth Symposium on Micro Machine and Human Science, pp. 39-43. IEEE Service Center, Piscataway, N7.

[5] Arcley D. H. A connectionist algorithm for genetic search // Proceedings of an Int. Conf. on Genetic Algorithms and Their Application. 1985, pp. 121-135.

[6] Goldberg D. E. Genetic Algorithms in Search, Optimization and Machine Learning. Reading, MA: Addision-Wesley, 1989.

[7] Gen M., Cheng R. Genetic Algorithms and Engineering Optimization. John Wiley and Sons, Inc. New-York, Chichester, Weinheim, Brisbane, Singapore, Toronto, 2000.

[8] Lazunov LA, Kureichik VM, Kureichik VV Genetic algorithms. - Rostov-on-Don: OOO, Rostizdat, 2004.

[9] Baabeau E., Dorigo M. and Theraulaz G. Swarm Intelligence: From National to Artificial Systems, New York, NY: Oxford University Press, Santa Fe Institute, Studies in the Science of Complexity, 1999. 
[10] Dorigo M. and Stutzle T. Ant Colony Optimization, MIT Press, 2004

[11] Cordon, "Herera and Stutzle" A Review on the Ant Colony Optimization Metcheuristic: Basis, Models and New Trends Mathware Soft Computing 9 (2002).

[12] 12. Shtovba S. D. Ant algorithms // Exponenta Pro. Mathematics in applications, 2003, No. 4, p. 70-75.

[13] Deb K. Genetic algorithms in multimodal function approximation. Master's thesis, University of Alabama, 1989.

[14] Parsopoules K. E., Vrahatis M. N. Recent approaches to global optimization problems through particle swarm optimization // Natural computing, 2002; 1 (2-3): 235-306.

[15] Abido M. A. Multi-objective particle swarm optimization for optimal power flow problem // 12 th International Middle East Power System Conference, 2008: 392-396.

[16] Baltar Alexandre M., Fontane Darell G. Use of multiobjective particle swarm optimization in water resources management// Journal of Water Resources Planning and Management, 2008: 257-265.

[17] Leong, Wen-Fung Yen, Garg G. Dynamic swarms in PSObased multi-objective optimization // IEEE Congress on Evolutionary Computation, 2007: 3172-3179.

[18] Andries P. Engelbrecht write, Ying Tan translate. Basic of computer swarm intelligence. Beijing: Qinghua Universitety Press, 2009.

[19] Dunwei Geng, Youn Zhang, Jianhua Zhang. Multi-objective particle swarm optimization based on minimal particle angle // Lecture Notes in Compute Science, 2005, 3644(1): 571-576.

[20] Yabin Wana, Jianmin Zhao, Xisheng Jia, Yan Tian. Spare parts allocation optimization in a multi-echelon support systems based on multi-objective particle swarm optimization method
// Eksploataciya i Niezawodnose - Maintenance and Reliability, 2014; 16(1): 29-36.

[21] Karimov N. A. The calculation of the spare parts in the autoservice enterprise on the base of actual demand // Engineering Science 78-84.

[22] Dyshin O. A., Karimov N. A. Identification of the actual distribution of demand for spare parts in car maintenance service stations // American Journal of Traffic and Transportation Engineering 26-31.

[23] Parsopdous R. E. and Vrahatis M. N. Particle swarm optimization method in multi-objective problems // Proceedings of the ACM 2002 Symposium on Applied Computing (pp. 603-607) ACM Press.

[24] Schaffer J. D. Multiple objective optimization with vector evaluated genetic algorithm // In Proceedings of the Ist International Conference on Genetic Algorithm (pp. 93-100). Morgan Raufmann Publishers, 1985.

[25] Sedighizadeh D., Masehian E. Particle System Optimization Methods, Taxonomy and Applications // International Journal of Computer Theory and Engineering, 2000, vol. 1, N5, 486$502 /$.

[26] Reyes-Sierra M. and Coello C. A. Multi-Objective Particle Swarm Optimizars: A Survey of the State-of0the-Art// International Journal of Computational Intelligence Research, 2006, vol. 2. N3, pp. 287-307.

[27] Zitzler E., Deb K. and Thiele L. Comparison of multiobjective evolution algorithms empirical results// Evolutionary Computation, 2000. 8(2): 173-195.

[28] Jin Y., Olhofer M. and Senfhoff B. Evolutionary dynamic weighted aggregation for multi-objective optimization: Why does it work and how? // Proceedings of the GECCO 2001 Conference, San Francisko, CA, pp. 1042-1049. 